\title{
A Phase-separating Thiol-ene Photoresin for Volumetric Bioprinting of Macroporous Hydrogels
}

Monica Zippora Müller, Robert W. Style, Ralph Müller, Xiao-Hua Qin*

M. Z. Müller, Prof. R. Müller, Dr. X.-H. Qin

Institute for Biomechanics, ETH Zurich

Leopold-Ruzicka-Weg 4, 8093 Zurich, Switzerland

*E-mail: qinx@ethz.ch

Dr. R. Style

Laboratory for Soft and Living Materials, ETH Zurich

Vladimir-Prelog-Weg 1-5/10, 8093 Zurich, Switzerland

Keywords: macroporous hydrogels, thiol-ene reaction, photopolymerization-induced phase separation (PIPS), volumetric printing, 3D cell culture 


\begin{abstract}
Macroporous hydrogels facilitate solute transport and cell-cell communication in 3D, but materials allowing for in situ pore formation and 3D-printing in aqueous solutions are scarce. Here, a phase-separating thiol-ene photoresin is developed for light-assisted 3D-printing of hierarchical macroporous hydrogels that support 3D cell growth. The resin consists of norbornene-functionalized polyvinyl alcohol, di-thiol crosslinker and dextran sulfate, which can rapidly form a hydrogel with interconnected pores by photopolymerization-induced phase separation (PIPS). The pore size is tunable in the range of $2-40 \mu \mathrm{m}$ as quantified by fast Fourier transformation of confocal imaging data and depends on light intensity, polymer composition and molecular charge. After 3D photoencapsulation, high cell viability $(>90 \%)$ is achieved and cell spreading in the macroporous hydrogels is significantly higher than in conventional nanoporous hydrogels. The incorporation of a thermo-reversible gelatin network enables its printability for tomographic volumetric bioprinting in the presence of human mesenchymal stem cells. A cm-scale cell-laden hydrogel construct with vasculature-like channels and interconnected pores can be 3D-printed in 12 seconds. The materials are cell-compatible, lowcost, easy-to-make and highly efficient for PIPS and light-based 3D-printing, which is unachievable with conventional 3D-printable hydrogels. Such materials are promising for future photofabrication of complex tissues with a hierarchical porosity within seconds.
\end{abstract}




\section{Introduction}

In the last decade, macroporous scaffolds ${ }^{1-3}$ have emerged as promising materials for $3 \mathrm{D}$ cell culture and tissue engineering since they facilitate solute transport and cell-cell communication. For instance, Sun et al. reconstructed 3D bone cell networks in vitro by culturing osteocytes within a bed of microbeads. ${ }^{4-5}$ However, the microbeads are non-degradable and not applicable for tissue implantation. Huebsch et al. created alginate-based macroporous hydrogels by adding hydrolytically degradable porogens for recruiting or releasing cells. ${ }^{6}$ Since hydrolytic degradation of porogens is a slow process, the embedded cells need at least 7 days to colonize and interact with the void space. Another method to create macroporous hydrogels is phase separation, which is especially interesting because of its easy fabrication, lack of harsh treatments and compatibility with direct cell encapsulation. Broguiere et al. used phaseseparating polyethylene glycol-based hydrogels to create 3D neuronal networks. ${ }^{7}$ However, this method necessitates a long crosslinking procedure (ca. 1 hour), and the materials are not 3Dprintable, making them unsuitable for fabrication of complex hydrogel constructs.

A major challenge in additive (bio)manufacturing ${ }^{8-10}$ is the shortage of bioresin formulations that allow digital fabrication of anatomically shaped hydrogel constructs with both macroscopically and microscopically controlled architecture to support cell infiltration, 3D cell growth and tissue regeneration. Existing hydrogel systems ${ }^{11-12}$ for 3D bioprinting often have very small pore sizes $(5 \mathrm{~nm}-50 \mathrm{~nm})$ that fail to provide a permissive environment for encapsulated cells. A combination of 3D printing and phase separation has been explored in other materials than hydrogels such as glass ${ }^{13}$ and acrylic monomers ${ }^{14-15}$ using digital light processing ${ }^{13,15}$ and two-photon polymerization. ${ }^{14}$ The problem with these materials is that the porogen needs to be removed by vacuum drying. Very recently, a photo-curable emulsion has been used for stereolithographic printing of porous ceramics. ${ }^{16}$ Yet, all these materials are unsuitable for 3D bioprinting of macroporous hydrogels in the presence of living cells. 
bioRxiv preprint doi: https://doi.org/10.1101/2022.01.29.478338; this version posted January 31, 2022. The copyright holder for this preprint (which was not certified by peer review) is the author/funder, who has granted bioRxiv a license to display the preprint in perpetuity. It is made available under aCC-BY-NC-ND 4.0 International license.

Herein, we report a method for 3D construction of macroporous hydrogels by leveraging stateof-the-art additive manufacturing with photopolymerization-induced phase separation (PIPS, Figure 1A). In PIPS, polymerization causes phase separation, driven by changes in entropy as monomers combine into polymer chains.
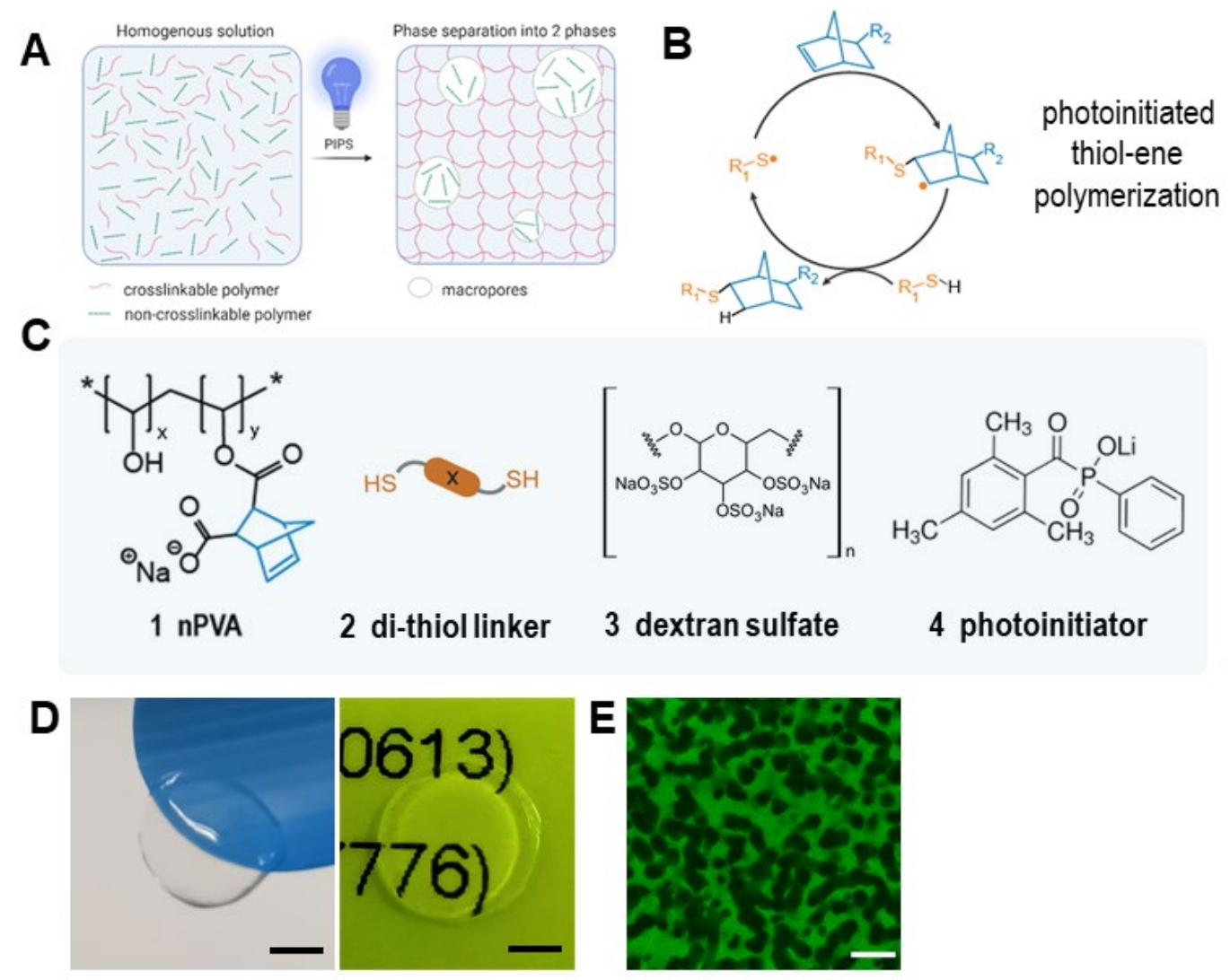

Figure 1: Macroporous hydrogels by photopolymerization-induced phase separation (PIPS). A) Schematic representation of the PIPS process: two initially miscible monomers become immiscible during the photocrosslinking of one monomer because of entropy change, which induces phase separation and pore formation. B) Schematic of radical-mediated photoinitiated thiol-ene polymerization. C) Chemical structures of key components for PIPS: 1) norbornene-functionalized polyvinyl alcohol (nPVA), 2) di-thiol linker, 3) dextran sulfate and 4) water-soluble photoinitiator (LAP, lithium phenyl-2,4,6-trimethylbenzoylphosphinate). C) Photographs of phase-separated hydrogels showing their optical transparency. Scale bars $=2 \mathrm{~mm}$. D) Representative confocal microscopy image of macroporous gels permeabilized with FITC-dextran. Scale bar $=10 \mu \mathrm{m}$.

For the first time, PIPS is used to produce biocompatible macroporous hydrogels within seconds via photoinitiated step-growth thiol-ene polymerization (Figure 1B). The key to this success is a novel phase-separating resin based on norbornene polyvinyl alcohol (nPVA), a di-thiol crosslinker, dextran sulfate (DS) and a photoinitiator (Figure 1C). The resin is easy-to-make, injectable, rapidly photocurable, modular and cell-compatible. ${ }^{12}$ After curing, the materials form stable hydrogels with a pore size in the range of 2-50 $\mu \mathrm{m}$ (Figure 1D-E) and can be 3D- 
printed by emerging biofabrication techniques such as tomographic volumetric photopatterning. ${ }^{9,18-19}$ This method holds great potential for new applications in 3D bioprinting of architected porous hydrogels for complex tissue engineering.

\section{Results \& Discussion:}

\subsection{Evidence of PIPS}

To determine potential compositions for interconnected pores, an approximate phase diagram of nPVA and DS was made (Figure 2A). It resembles a phase diagram of PVA and dextran found in literature. ${ }^{21}$ Assuming symmetry, the critical point was estimated to be at $2.5 \%$ DS and $2.5 \% \mathrm{nPVA}$. At this point, the volume contribution of both components is similar, so that interconnected pores are formed through spinodal decomposition. ${ }^{22}$ Further away from the critical point, nucleation and growth occurs. The binodal curve is the boundary between the regions where it is more favorable for the components to be mixed or phase separated. Below the binodal curve, the two polymers are miscible, while two separate phases exist in compositions above it. Most compositions were chosen just below the binodal curve, so that the material phase-separates during photopolymerization.

To evaluate the crosslinking ability of the chosen composition, photorheometry was performed. Photorheometry showed rapid crosslinking within 40 seconds, as indicated by the sharp increase of the storage modulus ( $\mathrm{G}^{\prime}$ ) after turning on the UV source (Figure 2B). The storage modulus after crosslinking was around $370 \mathrm{~Pa}$ (Mix 1 in Table 1).

To investigate if the composition phase-separates upon photopolymerization, fluorescently labelled nPVA was added to the formulation $\left(1 / 50^{\text {th }}\right.$ of non-labeled) as a reporter of phase separation. The samples before and after UV curing were investigated by confocal microscopy. As shown in Figure 2C, porous structures were observed only after UV curing. This observation confirms that the gel precursors were initially miscible and phase-separated during photoinduced crosslinking. Moreover, the pore morphology can be tuned by the DS 
concentration: $2.5 \%$ DS results in bi-continuous structures, while 3\% DS results in droplets. Radial intensity plots of the Fast Fourier Transformation (RIFFT) of the images after curing show a clear peak, implying a uniform length scale (Figure S1A). Both peaks correspond to a length scale of around $8 \mu \mathrm{m}$. With higher DS concentrations, the pores were inhomogeneous, which can be explained by phase separation before polymerization, as these compositions lie in the immiscible region of the phase diagram. No phase separation was found in the samples with lower DS concentrations and without DS (Figure S2).

Since the pore size may change dramatically after swelling, a dye diffusion experiment was performed to verify the gel microstructure. Confocal microscope imaging data showed that FITC-dextran $(M w, 500 \mathrm{kDa})$ could permeate the pore space (Figure 2D). The RIFFT showed a peak for both compositions (Figure S1B). No pores were seen in the sample without DS (Figure S3). The sample with 3\% DS had a length scale of around $8 \mu \mathrm{m}$. Surprisingly, the length scale for $2.5 \%$ DS was found to be around $3 \mu \mathrm{m}$, which is smaller than the length scale observed before swelling with the labelled nPVA. The porosity was around $40 \%$ and no significant difference was found between $2.5 \%$ and $3 \%$ DS. To summarize, the phase-separated PVA hydrogels are permeable by FITC-dextran and the pore size can be tuned by varying the DS concentration. 

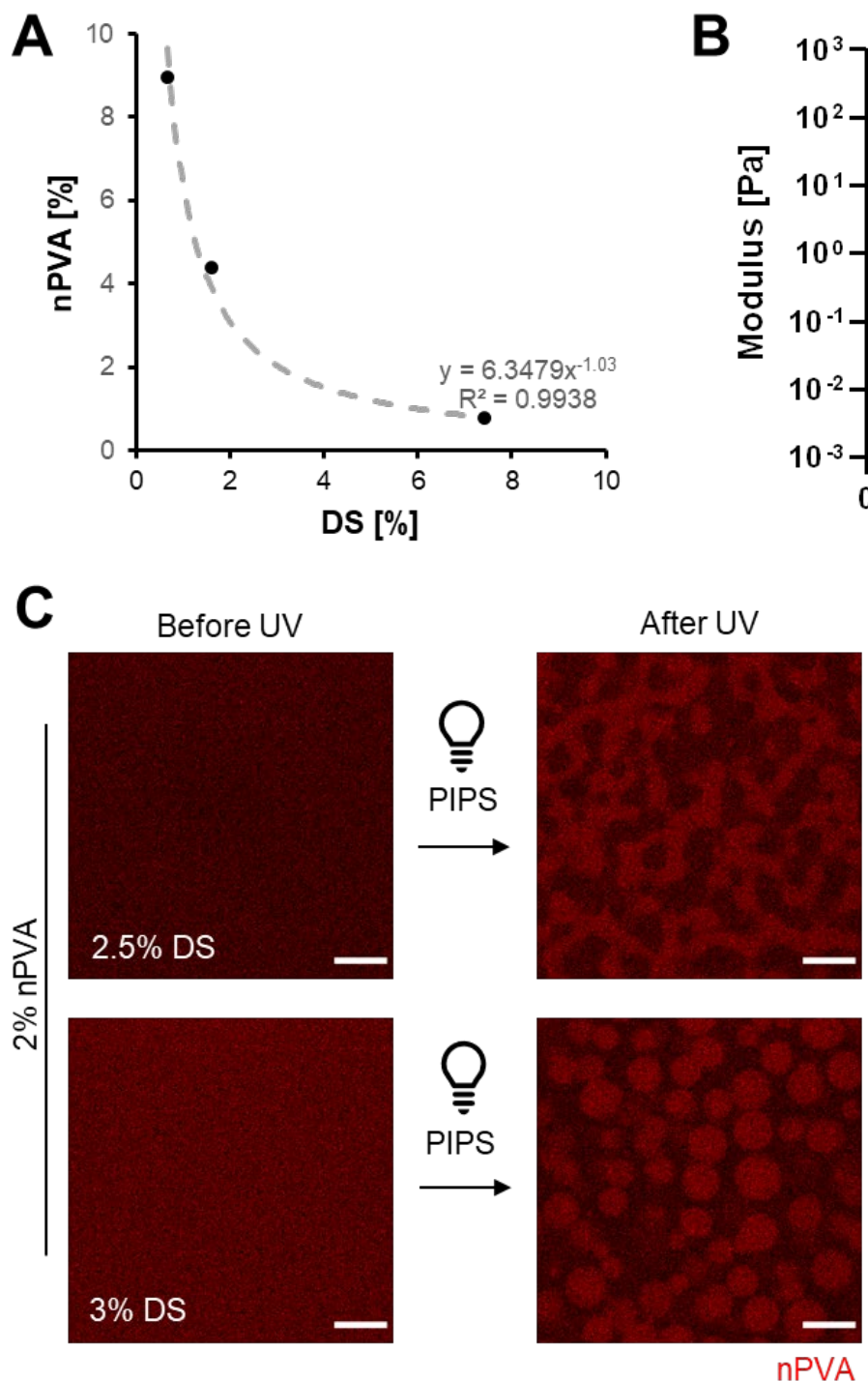

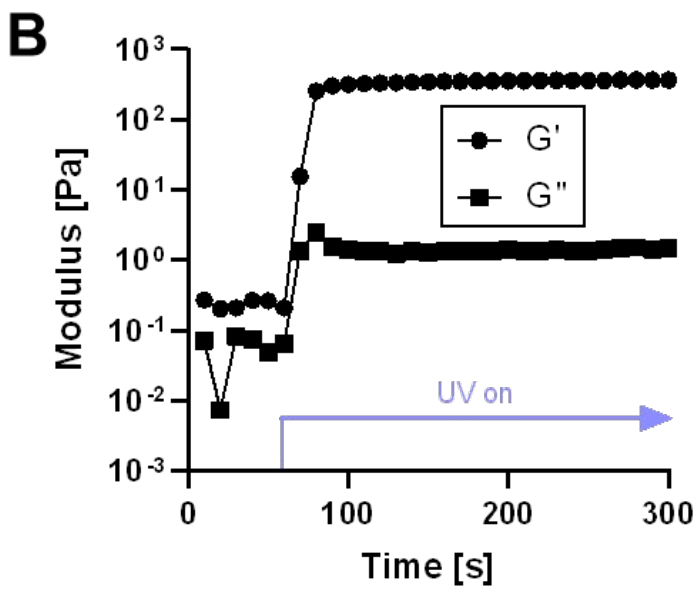

D

Figure 2: Macroporous hydrogels by PIPS between norbornene-functionalized PVA (nPVA) and dextran sulfates (DS). A) Approximation of a phase diagram at room temperature with nPVA (MW: $47 \mathrm{kDa}$ ) and DS (MW: $40 \mathrm{kDa})$. B) Time sweep of storage (G') and loss (G') modulus of Mix 1 (2\% nPVA 2.5\% DS) with an UV lamp $\left(236 \mathrm{~mW} \mathrm{~cm}^{-2}\right)$ turned on after $60 \mathrm{~s} ; 10 \mathrm{rad} \mathrm{s}^{-1}, 0.5 \%$ strain. C) Evidence of PIPS. Representative confocal images of Mix 1 and Mix 2 before and after UV crosslinking (18 $\left.\mathrm{mW} \mathrm{cm}^{-2}\right) .1 / 50$ th of FITC-labelled nPVA (red) was used for confocal imaging. D) Diffusion of FITC-dextran molecules into the pores. Representative confocal images of Mix $1(2.5 \% \mathrm{DS})$ and Mix $2(3 \% \mathrm{DS})$ crosslinked under UV irradiation $\left(18 \mathrm{~mW} \mathrm{~cm}^{-2}\right)$ after 3 days of swelling in PBS and then 2 days in a FITC-dextran solution (green, indicating the void space); images from middle of gels show that the dye has penetrated the hydrogel. Scale bars: $10 \mu \mathrm{m}(\mathrm{C}, \mathrm{D})$.

\subsection{Pore size, crosslinking and turbidity can be tuned by the composition}

To study the effect of molecular charge on PIPS, the phase-separating resin was compared to a composition with the uncharged dextran instead of DS. With the same concentration, no pores were seen in the sample with dextran while pores were seen with DS (Figure 3A). RIFFT analysis revealed that there is no peak in the sample with dextran, whereas a peak corresponding 
to a pore size of $5 \mu \mathrm{m}$ were found in the sample with DS. These results imply that charged molecules promote pore formation and highlight the uniqueness of negatively charged DS for PIPS, which promotes pore formation through repelling the negatively charged nPVA.

Resins with a higher nPVA content were explored as alternative compositions. A resin containing 3\% nPVA and 3\% DS exhibited larger pores after PIPS (Figure 3B) with the peak of the RIFFT corresponding to a length scale of $35 \mu \mathrm{m}$. With $2.5 \% \mathrm{DS}$, pores on a length scale of $4 \mu \mathrm{m}$ were observed. At $3.5 \% \mathrm{DS}$, the composition probably phase-separated before polymerization (Figure S4). Compositions with higher nPVA concentrations were also explored, but the pores were either not interconnected or less homogenously distributed, probably because of phase separation before photopolymerization (Figure S5).

Photo-rheology was used to determine if physical properties of the hydrogels can be tuned by the composition. The storage modulus was higher with increase of nPVA with the same thiolene ratio among groups (Figure 3C-D). The mean values were 146, 1'344 and 3'186 Pa for 2, 3 and 5\% nPVA, respectively. These data indicate the increase of crosslinking density as the increase of polymer content. Notably, the photo-curing of a resin with $1 \%$ nPVA and $2.5 \%$ DS was unsuccessful due to insufficient crosslinking and too low polymer content.

Next, we tested if the variation of DS concentration leads to changes in gel stiffness (defined as the $\mathrm{G}^{\prime}$-plateau). Interestingly, the stiffness was significantly higher for the sample with $2.5 \%$ DS compared to the control without DS (Figure 3E). This is likely due to the effect of partitioning where nPVA and PEG-2-SH as crosslinker may be rich in one phase whereas DS is rich in the other phase. This may lead to a higher crosslinking density in the nPVA-rich phase, which results in a higher bulk stiffness. This is supported by no significant difference between $0 \%$ and $2 \%$ DS gels, which both did not show signs of phase separation based on confocal imaging data (data not shown). The amplitude and frequency sweeps showed that a strain of 
bioRxiv preprint doi: https://doi.org/10.1101/2022.01.29.478338; this version posted January 31, 2022. The copyright holder for this preprint (which was not certified by peer review) is the author/funder, who has granted bioRxiv a license to display the preprint in perpetuity. It is made available under aCC-BY-NC-ND 4.0 International license.

$0.5 \%$ was within the linear viscoelastic region and the gels have a solid behavior over a wide range of frequencies (Figure S6).

A

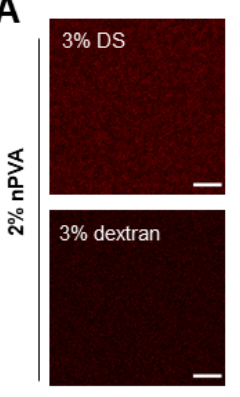

B

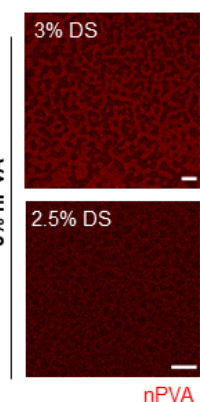

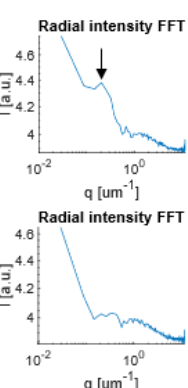

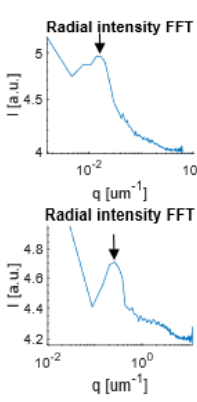

C

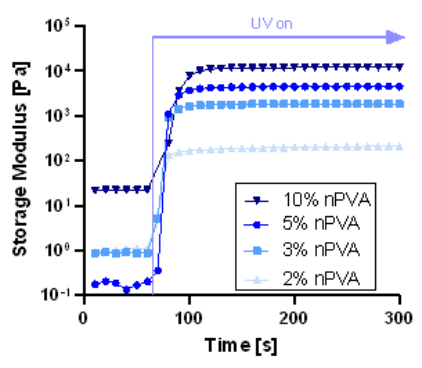

$\mathbf{F}$

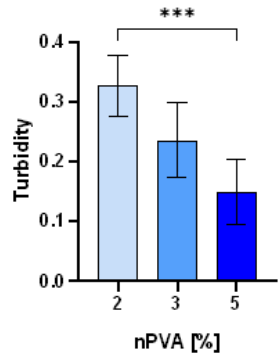

D

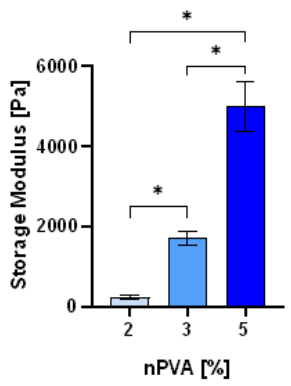

G

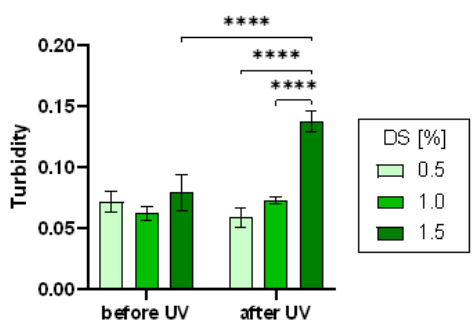

E

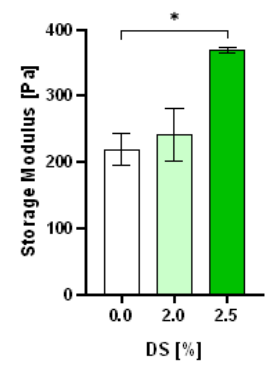

[\%]

Figure 3: The effect of material composition on structural and physical properties of PVA hydrogels. A) Molecular charge promotes the phase separation between nPVA and dextran derivatives. Representative confocal images (left) of Mix 3 containing 2\% nPVA and 3.1\% dextran derivatives after UV curing $\left(18 \mathrm{~mW} \mathrm{~cm}^{-2}\right)$. A 1/50th of FITC-labelled nPVA (red) was used for confocal imaging. RIFFT of the images showing a clear peak corresponding to a length scale of $5 \mu \mathrm{m}$ (arrow) with DS but not with dextran. B) Phase separation with $3 \% \mathrm{nPVA}$ and different DS concentrations resulting in pores at different scales. Representative confocal images after UV curing $\left(18 \mathrm{~mW} \mathrm{~cm}^{-2}\right)$. RIFFT of the images showing clear peaks (arrows) corresponding to a length scale of $35 \mu \mathrm{m}$ (top) and $4 \mu \mathrm{m}$ (bottom), respectively. C-E) In situ photorheometry of hydrogels with varying nPVA (C-D) and DS (E) concentrations; all measured with $10 \mathrm{rad} \mathrm{s}^{-1}$ and $0.5 \%$ strain in humidified atmosphere. C) Representative time sweep of storage moduli (G') of gels with $2 \%$ DS and varying nPVA with UV lamp $\left(236 \mathrm{~mW} \mathrm{~cm}^{-2}\right)$ turned on after 60 s. D-E) G' of gels with either 2\% DS (D) or 2\% nPVA (E) after 4 min of crosslinking (N=3); BrownForsynthe \& Welch one-way ANOVA with Dunnet's multiple comparisons test, $p$-values: $p=0.0102$ ( 2 vs. $3 \%$ nPVA), $\mathrm{p}=0.0115$ ( $2 \%$ vs. $5 \%$ nPVA), $\mathrm{p}=0.0258$ ( $3 \%$ vs. $5 \% \mathrm{nPVA}), \mathrm{p}=0.0177$ ( $0 \%$ vs. $2.5 \%$ DS). F-G) Gel turbidity is higher in phase-separated compositions close to the critical point $(\mathrm{N}=5)$. F) Turbidity of UV-cured (18 $\mathrm{mW} \mathrm{cm}{ }^{-2}$ ) nPVA gels with $2.5 \%$ DS in PBS showing significantly higher turbidity with $2 \% \mathrm{nPVA}$ compared to $5 \%$ nPVA; ordinary one-way ANOVA followed by Tukey's multiple comparisons test, $* * *: p=0.0009 . \mathrm{G}$ ) Turbidity of $4 \% \mathrm{nPVA}$ gels with $0.5,1$ and $1.5 \%$ DS before and after UV curing $\left(20 \mathrm{~mW} \mathrm{~cm}{ }^{-2}\right)$; two-way ANOVA followed by Tukey's multiple comparisons test, ${ }^{* * * *}: \mathrm{p}<0.0001$. Scale bars: $10 \mu \mathrm{m}$ (A, B bottom image) and 50 $\mu \mathrm{m}$ (B top image). Data presented as mean \pm SD.

The influence of gel composition on turbidity of the hydrogels was studied on a plate reader.

The turbidity was higher in hydrogels with compositions close to the critical point. For instance,

hydrogels with $2 \%$ nPVA and $2.5 \%$ DS showed a significantly higher level of turbidity compared to 5\% nPVA gels with the same DS concentration (Figure 3F). Additionally, the turbidity of formulations with 4\% nPVA and varying DS before and after UV curing was measured. Before UV curing, there is no significant difference in the turbidity. After UV curing, 
the turbidity of hydrogels with $1.5 \%$ DS was significantly higher compared to $0.5 \%$ DS and $1 \%$ DS groups as well as all groups before UV curing (Figure 3G). This indicates that the composition with 4\% nPVA and $1.5 \%$ DS is initially miscible, but phase-separates upon polymerization.

Table 1: Composition and properties of common mixes used in this study.

\begin{tabular}{|c|c|c|c|c|c|c|}
\hline Name & $\begin{array}{c}\text { nPVA \% } \\
(w / w)^{a}\end{array}$ & $\begin{array}{l}\text { DS \% } \\
\text { (w/w) }\end{array}$ & $\begin{array}{l}\text { Pore sizes } \\
{[\mu \mathrm{m}]}\end{array}$ & $G^{\prime}[\mathrm{Pa}]$ & G' [Pa] & G'/G' \\
\hline Mix 0 & 2.0 & - & $N A^{d}$ & $220 \pm 24$ & $1.42 \pm 0.34$ & $0.007 \pm 0.002$ \\
\hline Mix 1 & 2.0 & 2.5 & $8 / 3$ & $370 \pm 4$ & $1.51 \pm 0.09$ & $\begin{array}{l}0.004 \pm \\
0.0002\end{array}$ \\
\hline Mix 2 & 2.0 & 3.0 & $8 / 7$ & $\begin{array}{l}\text { not } \\
\text { measured }\end{array}$ & $\begin{array}{l}\text { not } \\
\text { measured }\end{array}$ & $\begin{array}{l}\text { not } \\
\text { measured }\end{array}$ \\
\hline Mix 3 & 2.0 & 3.1 & $2-8 / N / A$ & $\begin{array}{l}\text { not } \\
\text { measured }\end{array}$ & $\begin{array}{l}\text { not } \\
\text { measured }\end{array}$ & $\begin{array}{l}\text { not } \\
\text { measured }\end{array}$ \\
\hline $\operatorname{Mix} 4^{c}$ & 2.0 & 1.0 & N/A / 18 & $\begin{array}{l}4249 \pm \\
1063^{e}\end{array}$ & $94.2 \pm 38.9^{\mathrm{e}}$ & $\begin{array}{l}0.022 \pm \\
0.004^{\mathrm{e}}\end{array}$ \\
\hline
\end{tabular}

${ }^{a}$ Weight concentration was used to correlate with the approximated phase diagram; ${ }^{\mathrm{b}}$ determined by RIFFT of confocal imaging data, without/with swelling; ${ }^{\mathrm{c}} 5 \%$ gelatin was added to enhance the viscosity of photoink; ${ }^{\mathrm{d}}$ no pores detected; ${ }^{\mathrm{e}}$ before incubation at $37{ }^{\circ} \mathrm{C}$ (prior to gelatin release). DS (MW: 40 $\mathrm{kDa}), 0.05 \%$ LAP, PEG-2-SH crosslinker for a thiol:ene ratio at 4:5.

\subsection{High Light Intensity Leads to Smaller Pores and Accelerates Crosslinking}

Tuning the pore size by light intensity is an important criterion for the applicability of these phase-separating inks for light-based 3D printing. Such tuning has been shown in purely organic polymeric matrices in literature, ${ }^{23}$ but no previous studies have demonstrated tuning of porosity in a hydrogel system. Thus, the effect of light intensity $\left(5-100 \mathrm{~mW} \mathrm{~cm}^{-2}\right)$ on PIPS and pore size distribution was investigated. As anticipated, imaging of gels cured at different light intensities showed that the length scale of the pores can be tuned by the UV light intensity (Figure 4A). For irradiation at an intensity of $5 \mathrm{~mW} \mathrm{~cm}^{-2}$, the pore size was $8 \mu \mathrm{m}$. As the light intensity is increased to $10 \mathrm{~mW} \mathrm{~cm}{ }^{-2}$, the pore size decreased to $5 \mu \mathrm{m}$. Further, it decreased to $2 \mu \mathrm{m}$ when increasing the light intensity further to $100 \mathrm{~mW} \mathrm{~cm}{ }^{-2}$ (Figure 4B). The best fit was obtained with the following function: 
A

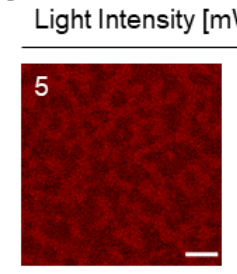

C

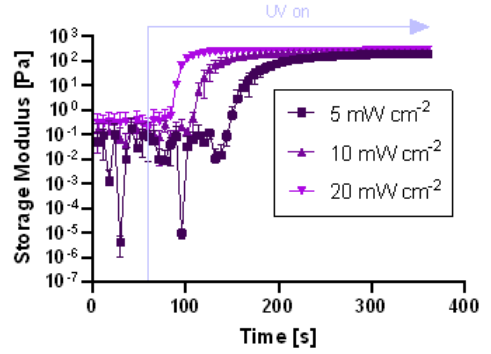

\section{0}

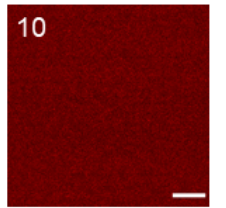

D

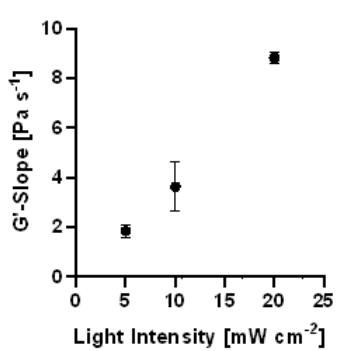

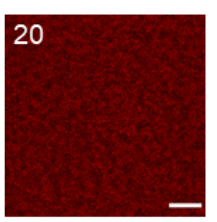

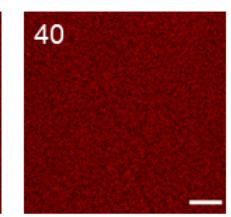

$\mathbf{E}$

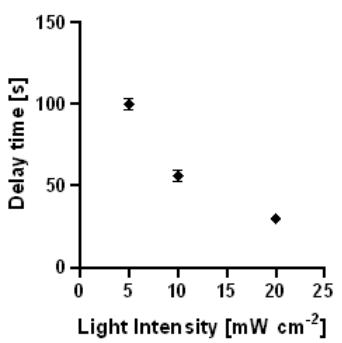

B

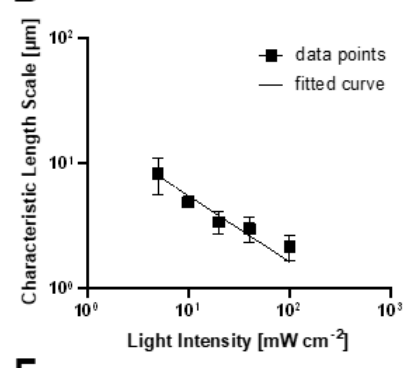

$\mathbf{F}$

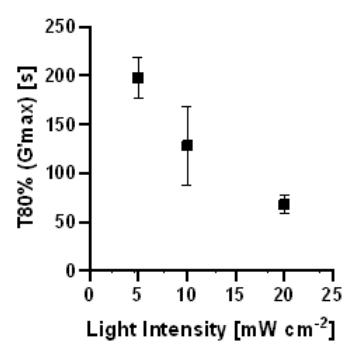

Figure 4: The effect of light intensity on pore size and crosslinking dynamics. A-B) Characteristic length scale of the pores can be tuned as a function of the light intensity. A) Representative confocal images of UV-cured hydrogels (Mix 3) with light intensities ranging from 5 to $100 \mathrm{~mW} \mathrm{~cm}^{-2}$. B) Characteristic length scale obtained from RIFFT plot peaks of such images $(\mathrm{N}=3)$; curve fit with a power function. $\mathbf{C}-\mathrm{F})$ Crosslinking dynamics with different light intensities: effect on the time sweep of gel storage moduli (C) and G'-slope (D), delay time for crosslinking (E) and time to reach $80 \%$ of the G'-plateau $(\mathrm{F})$ under UV irradiation from $60 \mathrm{~s}$ at different light intensities $\left(5-20 \mathrm{~mW} \mathrm{~cm}^{-2}\right)$. Data presented as mean \pm SD. Scale bars $=10 \mu \mathrm{m}(\mathrm{A})$.

Photorheometry showed that an increase in light intensity accelerates crosslinking (Figure 4C-

F). The evolution of G' with different UV light intensities is shown in Figure 4C. For analyzing

the crosslinking speed, a linear regression at the section where the $\mathrm{R}^{2}$ exceeds 0.99 was made as described elsewhere. ${ }^{24}$ The slope, which indicates the crosslinking speed, was higher with increased light intensity (Figure 4D). The delay time denotes the timepoint where the linear regression curve starts (Figure $4 \mathrm{E}$ ), whereas $\mathrm{T}_{80} \%$ refers to the time to reach $80 \%$ of the maximum G' within $5 \mathrm{~min}$ of UV curing. When increasing the light intensity from $5 \mathrm{~mW} \mathrm{~cm} \mathrm{c}^{-2}$ to $20 \mathrm{~mW} \mathrm{~cm}^{-2}$, the crosslinking speed is increased from $1.8 \mathrm{~Pa} \mathrm{~s}^{-1}$ to $8.8 \mathrm{~Pa} \mathrm{~s}^{-1}$, whereas the delay time is decreased from $100 \mathrm{~s}$ to $30 \mathrm{~s}$, and the time to reach $\mathrm{T}_{80 \%}$ is decreased from $198 \mathrm{~s}$ to $68 \mathrm{~s}$, respectively. The results confirmed that photocrosslinking is faster at higher light intensities (Figure 4F). 
bioRxiv preprint doi: https://doi.org/10.1101/2022.01.29.478338; this version posted January 31, 2022. The copyright holder for this preprint (which was not certified by peer review) is the author/funder, who has granted bioRxiv a license to display the preprint in perpetuity. It is made available under aCC-BY-NC-ND 4.0 International license.

Together, these findings suggest that the light intensity can be used to tune the crosslinking dynamics and PIPS, which influences the length scale of the pores. Kimura et al. reported phase separation induced by photopolymerization in a mixture of polystyrene and methylacrylate. ${ }^{25}$ By changing the light intensity, a variety of stationary morphologies were obtained. With higher light intensity, the morphology has less time to evolve before it is arrested and frozen at the onset of the gelation.

\subsection{Cellular Behavior in Phase-separating Hydrogels}

For studying cellular behavior in the phase-separating gels, human mesenchymal stem cells (hMSCs) were encapsulated in the hydrogel. The cells were mixed with the hydrogel precursors and cured under a UV lamp (Figure 5A). Phase-separating compositions with 2.5 and 3\% DS showed a significantly higher viability ( $>90 \%$ ) compared to the control without DS (Figure 5BC). The $2.5 \%$ DS group even showed an excellent viability ( $>95 \%)$ on average.

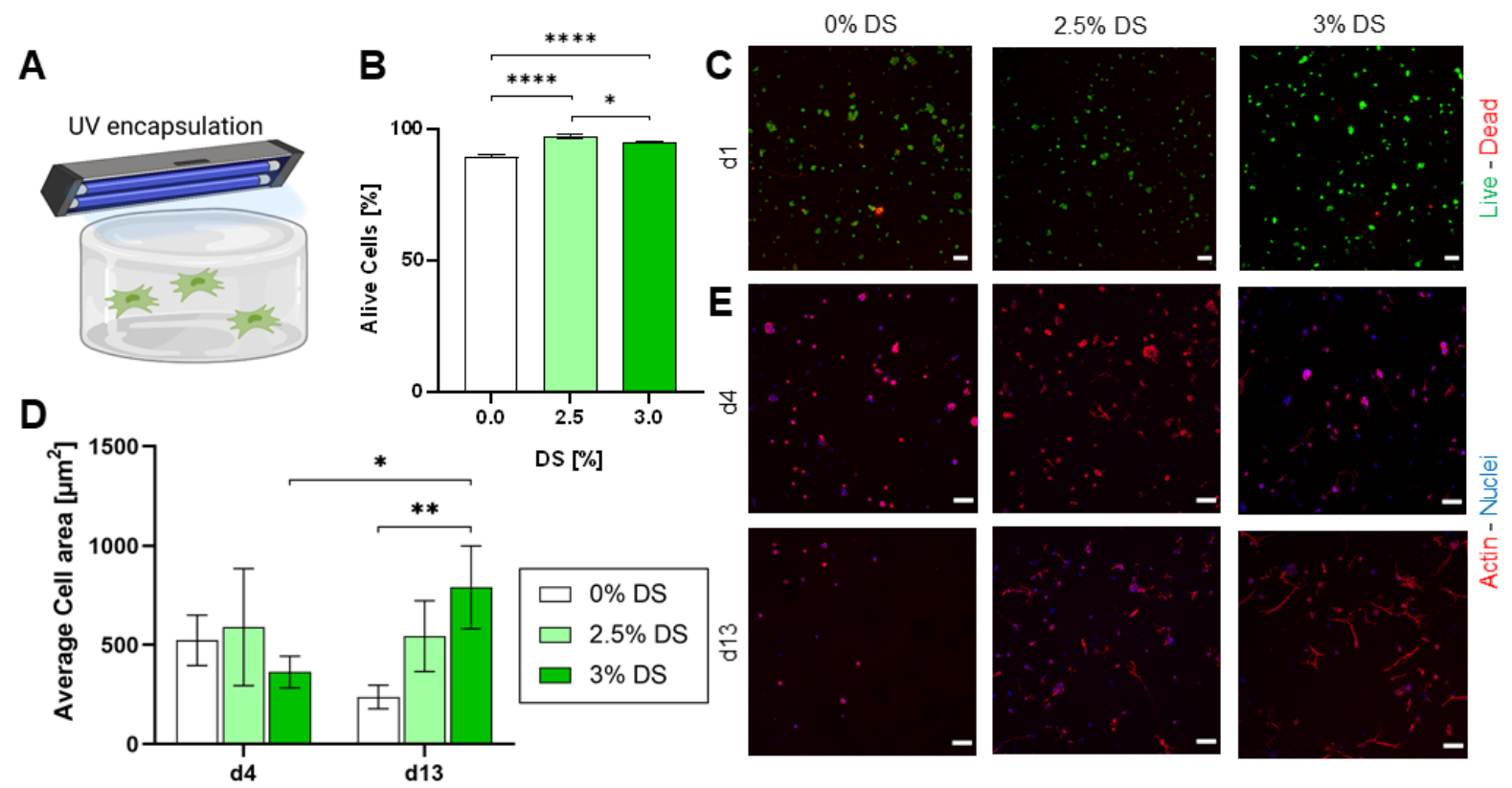

Figure 5: 3D Photoencapsulation of hMSCs in macroporous PVA hydrogels. A) Schematic representation of UV cell encapsulation, where the gel is crosslinked under UV in the presence of live hMSC cells. B) Cell viability on day 1 as determined by a live-dead assay $(\mathrm{N}=3)$; one-way ANOVA with Tukey's correction for multiple comparisons, $*: \mathrm{p}=0.0180, * * * * \mathrm{p}<0.0001 . \mathrm{C}$ ) Representative confocal images of live-dead (green-red) stained hMSCs on day 1, maximum intensity projections (MIP) from z-stacks $(100 \mu \mathrm{m})$ : light intensity at $20 \mathrm{~mW} \mathrm{~cm}^{-2}, 5$ min. D-E) Cell morphology in nPVA hydrogels. D) Average cell area after 4 and 13 days of 3D osteogenic culture $(\mathrm{N}=3)$; two-way ANOVA with correction for multiple comparisons with Sidak for comparison of the two timepoints and with Tukey for comparisons of the three compositions, *: $p=0.0358, * *: p=0.0063$. E) Representative confocal images of actin-nuclei-stained cells. Data presented as mean \pm SD. Scale bars $=100 \mu \mathrm{m}$ $(\mathrm{A}, \mathrm{E})$ and $20 \mu \mathrm{m}(\mathrm{D})$. 
The phase-separating compositions also showed a higher average cell area compared to the control. Cells in 3\% DS gels showed a significantly higher cell area compared to the control without DS on day 13 (Figure 5D). Further, the cell area significantly increased over time. Similarly, the images of the actin-nuclei stained cells showed longer dendrites in the phaseseparating gels (Figure 5E). The dendrite length increases over time in the phase-separating compositions and seems to be higher in 3\% DS than in 2.5\% DS gels, which can be explained by the larger pore size of 3\% DS gels (Figure S7). Dendrites penetrating the pores were also observed (Figure S8).

\subsection{Volumetric Bioprinting with An Optimized Resin}

The phase-separating resins were combined with the Readily3D tomographic volumetric printer, which allows rapid construction of 3D living hydrogel constructs in a rotating glass vial within seconds (Figure 6A). Initial printing attempts were unsuccessful due to insufficient viscosity of the resins. Without additional components, no successful polymerization was observed in a laser dose test. Instead of polymerized dots, streaks were found, which may mean that the material was partially polymerized but sedimented. In volumetric printing, a viscous or physically crosslinked material is often needed. Gelatin was added to the composition as a viscosity enhancer.

A resin containing 2\% nPVA, 1\% DS, and 5\% gelatin (Mix 4, Table 1) showed homogenous distribution of porosity. With higher DS concentrations of $2 \%$ DS and above, gels were not transferrable after washing, which may be explained by premature phase separation of gelatin with DS, as gelatin and dextran have been found to phase separate. ${ }^{26}$ Unwanted phase separation was seen with $1.5 \%$ DS, while no phase separation was seen with $0.5 \%$ DS (Figure S9). With $1 \% \mathrm{DS}$, pores in the range of about 2-5 $\mu \mathrm{m}$ were observed (Figure S10).

Further, the composition showed temperature-dependent gelation and a viscosity higher than water. A temperature sweep from $37{ }^{\circ} \mathrm{C}$ to $8{ }^{\circ} \mathrm{C}$ showed a gelation point at around $20-22{ }^{\circ} \mathrm{C}$ (Figure S11A). Above $22^{\circ} \mathrm{C}$, the complex viscosity was about $200 \mathrm{mPa} \cdot \mathrm{s}$ (Figure S11B), which 
bioRxiv preprint doi: https://doi.org/10.1101/2022.01.29.478338; this version posted January 31,2022. The copyright holder for this preprint (which was not certified by peer review) is the author/funder, who has granted bioRxiv a license to display the preprint in perpetuity. It is made available under aCC-BY-NC-ND 4.0 International license.

is roughly 200 times higher than the viscosity of water and almost seven times higher than the composition without gelatin. Crosslinking the composition at $25^{\circ} \mathrm{C}$ is indicated by a clear increase in G' and G', after UV irradiation for $24 \mathrm{~s}$ (Figure S11C). A plateau in G' of about 2 $\mathrm{kPa}$ was reached within less than one minute. The elastic modulus $\mathrm{G}^{\prime}$ is much higher compared to compositions without gelatin.

Laser dose tests were performed to determine a suitable laser dose range for the resin. An estimate of $140 \mathrm{~mJ} \mathrm{~cm}^{-2}$ was made with a first dose test (Table S1). Then, a second test with smaller intervals was performed and a threshold of $75 \mathrm{~mJ} \mathrm{~cm}^{-2}$ found (Table S2). 3D objects could be printed with a laser dose in the range of 95 to $125 \mathrm{~mJ} \mathrm{~cm}^{-2}$. The volumetric prints and laser dose tests were conducted above room temperature because the ink turned turbid at room temperature, which may affect light penetration and printability. The resin remained as a viscous liquid during printing. The turbidity is presumably due to phase separation between gelatin and other components such as DS, which may occur when gelatin is physically crosslinked. At $90 \mathrm{~mJ} \mathrm{~cm}^{-2}$ and below, a turbid structure was visible towards the end of the print but disappeared when swirling the vial. This observation can be explained by PIPS: as the material is polymerizing, phase separation is initiated because of a decrease in entropy even before the gel point is reached. When swirling, the phase-separated liquid resin is mixed with unpolymerized resin, leading to a lower concentration of polymerized material.

Using volumetric printing, a vascular branch model was printed at a laser dose of $95 \mathrm{~mJ} \mathrm{~cm}^{-}$ ${ }^{2}$ within 12 seconds (Supplementary Movie M1). The model (Figure 6B) has a height of 1.4 $\mathrm{cm}$, vascular channels with dimensions of $0.7-1.4 \mathrm{~mm}$. The turbid construct is depicted while printing (Figure 6C). Channels were shown to be perfusable after leaching out the gelatin for two days at $37^{\circ} \mathrm{C}$ and subsequent incubation in a FITC-dextran solution for one day (Figure 6D). The printing time is 12 seconds. Printing at $95 \mathrm{~mJ} \mathrm{~cm}^{-2}$ led to ultrasoft constructs that are difficult to transfer, whereas prints at higher doses such as $125 \mathrm{~mJ} \mathrm{~cm}^{-2}$ resulted in stiffer constructs. 


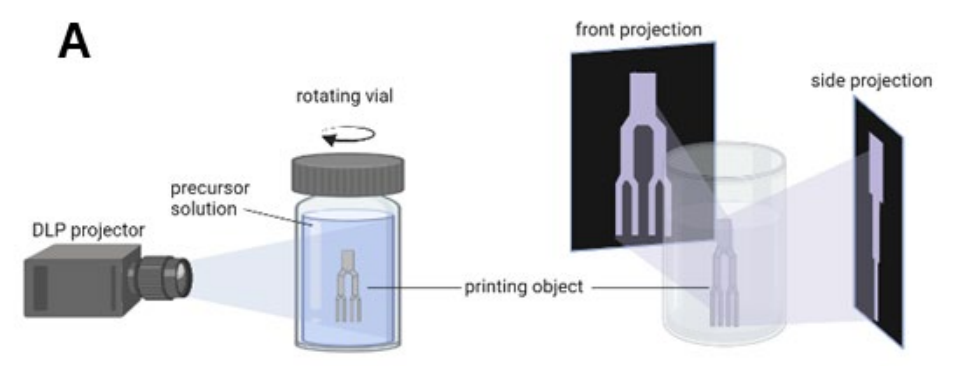

B
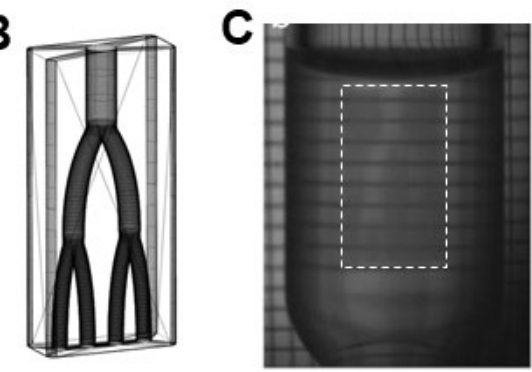

$\mathbf{F}$

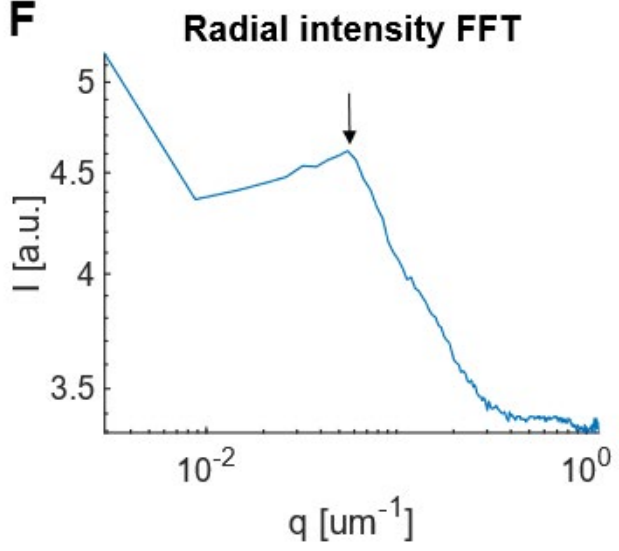

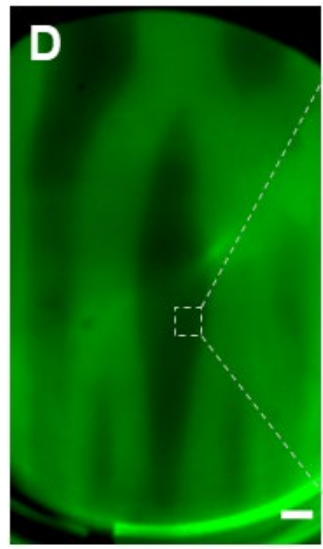

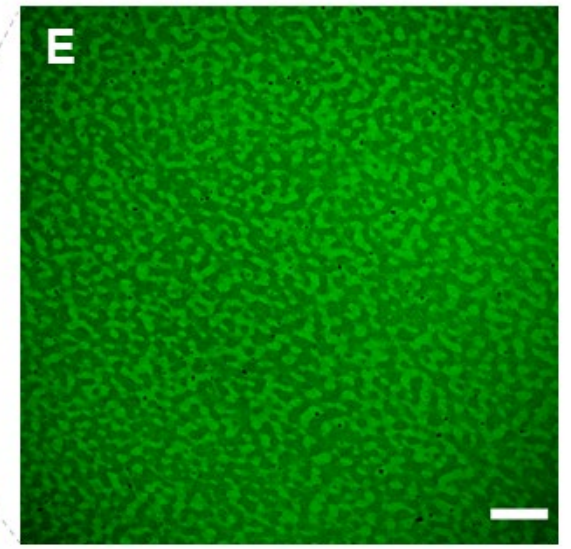

FITC-dextran

Figure 6: Volumetric tomographic photofabrication of macroporous hydrogels using the phase-separating ink. A) Schematic representation of the ultrafast volumetric bioprinting technique: a cm-scale, anatomic shaped 3D object can be fabricated within 10-30s in a rotating vial containing the photoink solution while tomographic light patterns of the 3D model are projected into the volume from multiple angles using a DLP modulator. B) STL model of a vascularized branch model. C) Photograph of the sample during the volumetric photofabrication process with the printed object marked by a rectangle. D) Stereomicroscope image of the printed structure after two days storage in PBS at $37^{\circ} \mathrm{C}$ to leach out gelatin and one day in FITC-dextran solution (green). E) Representative confocal image at the border of the same construct after two days in FITC-dextran. F) RIFFT of the image shown in shown in E) showing a clear peak (arrow) indicating a uniform length scale of $18 \mu \mathrm{m}$ for the porosity. Scale bars: $1 \mathrm{~mm}$ (D) and $50 \mu \mathrm{m}(\mathrm{E})$.

Next, we investigated if the printed constructs are indeed macroporous. After two days incubation in PBS at $37^{\circ} \mathrm{C}$ for gelatin release and another two days in a solution of FITCdextran, confocal imaging data showed interconnected pores in the printed gel constructs (Figure 6E). The RIFFT of this image showed a clear peak corresponding to characteristic length scale of around $18 \mu \mathrm{m}$ close to the border (Figure $6 \mathrm{~F}$ ) and $17 \mu \mathrm{m}$ in the middle of the gel (see Figure S12). The background signal was lower when imaging at the border of the construct after incubation in FITC-dextran at $4{ }^{\circ} \mathrm{C}$ for one day without prior incubation in PBS at $37{ }^{\circ} \mathrm{C}$ (Figure S13). This observation may be because the dye can penetrate the PVA hydrogel if given more time to diffuse. 
An initial experiment showed that the branch structure could be printed in the presence of live hMSCs. After bioprinting, the channels were perfusable by phenol red stained medium (Figure

S14A). The gel appeared to be macroporous (Figure S14B). Cells appeared highly viable as evidenced by a Calcein-AM staining, but further work is needed to study cell activity after printing (Figure S14B-C).

\section{Conclusion}

In conclusion, a novel phase-separating photoresin for light-based 3D bioprinting was developed. Further, the pore size could be fine-tuned by the light intensity. The newly developed phase-separating nPVA gels showed a high viability on day one and increased spreading over two weeks. First successes were shown with volumetric bioprinting, where a $\mathrm{cm}$-scale construct with macropores could be printed in only $12 \mathrm{~s}$. To the best of our knowledge, the present study is the first report of PIPS for 3D printing of cell-compatible macroporous hydrogels.

The developed phase-separating resins could be applicable to several fields of research. Although volumetric printing is most suitable for printing complex structures of clinically relevant sizes, the developed bioresin could also be used for a variety of other light-based 3D printing techniques such as digital light processing. Even the use in extrusion bioprinting is foreseeable, as gelatin is thermo-responsive. ${ }^{27}$ In the case of light-based $3 \mathrm{D}$ printing, different pore sizes may be digitally 3D-printed within one object by tuning the light intensity and PIPS. Accordingly, complex hierarchical structures with macropores could be generated in the presence of living cells, which could significantly advance the fields of biofabrication and functional tissue engineering. 


\section{Experimental Section}

\section{$\underline{\text { Materials }}$}

A final concentration of $0.05 \%$ LAP was used throughout all experiments with UV curing. nPVA (47kDa, DoF 7\% unless otherwise mentioned) was synthesized as described elsewhere. ${ }^{28}$ It was used at different concentrations and dissolved in a solution containing the photoinitiator by vortexing and 15 - 30 min of ultrasonication. PEG-2-SH ( $2 \mathrm{kDa}$ unless otherwise mentioned, LaysanBio) was used as a crosslinker with a concentration that results in a thiol/ene ration of 0.8. It was dissolved just before mixing in PBS with a $\mathrm{pH}$ of 7.4 or, in the case of longer experiments, with a pH of 6.05 for better stability. DS (40 kDa, Carl Roth) was used for phase separation and was dissolved in PBS at $50^{\circ} \mathrm{C}$ for one to two hours with vortexing every half hour. Dextran (40 kDa, Sigma-Aldrich) was dissolved similarly. In cellular experiments, CGRGDSP (China Peptides) was added for cell attachment. All components were dissolved in PBS.

\section{Approximation of a Phase Diagram}

An approximation of a phase diagram for the nPVA with DS using a "mini-titration setup" was made. In a small glass vial, about $1 \mathrm{~mL}$ of a $10 \%(\mathrm{w} / \mathrm{v}) \mathrm{nPVA}$ solution was prepared. $200 \mu 1$ from this were transferred to a new glass vial. While stirring this nPVA solution with a small magnetic stirrer $(8 \times 3 \mathrm{~mm})$, the dextran $(20 \% \mathrm{w} / \mathrm{v})$ or DS $(50 \% \mathrm{w} / \mathrm{v})$ solution was added dropwise with less than $100 \mu$ drops until the solution turned visibly turbid (without turbidity disappearing), similar as described in literature ${ }^{17}$. The tube containing the DS/dextran solution was weighed before and after titration. The same was done also with lower nPVA concentrations of $5 \%$ and $1 \%(\mathrm{w} / \mathrm{v})$. The receiving nPVA vial was weighed before and after titration to minimize any error caused by the retention of dextran on the pipette tip. For the phase diagram, the concentrations were all calculated to $\mathrm{w} / \mathrm{w}$. 


\section{Hydrogel Preparation}

Thorough mixing is essential for phase separation and was ensured by pipetting up and down 20 times after the addition of each component. When the volume was large enough, e.g. in volumetric printing experiments, the composition was mixed until it turned visibly clear or homogenous. In some cases, additional vortexing for two to three seconds was performed. In case of bubbles and small sample volumes, the tube was centrifuged for a few seconds and then vortexed again. The crosslinker was usually added last. Detailed descriptions on the hydrogel preparations can be found in the chapters of each composition.

\section{nPVA-DS Phase-Separating Composition}

The composition for Mix 1 is described in detail in Table S3. The components were added in the order listed, where it was especially important that the crosslinker is added last. For cell embedding, the order of mixing was altered to minimize the time where cells are outside of their medium (Table S4).

\section{The Bioresin Based on nPVA-DS-Gelatin}

The addition of gelatin was inspired by a study showing its use to transiently stabilize bioresins in extrusion bioprinting. ${ }^{27}$ Gelatin was explored mainly because it gelates at lower temperatures, which makes it suitable to prevent sedimentation in volumetric printing. Gelatin was dissolved at $40^{\circ} \mathrm{C}$ in the waterbath for two to three hours for a stock solution of $10 \%(\mathrm{w} / \mathrm{v})$. The composition used for printing experiments in the end is described in Table S5. The components were mixed in this order and were warmed to prevent gelation during mixing. Alone the PEG2-SH was not warmed to keep it stable. Cells were suspended in the gelatin portion.

\section{Rheometry}

In most experiments, a modular Anton Paar MCR 302 photo-rheometer was used. A glass plate and parallel plate with $20 \mathrm{~mm}$ diameter (PP20) were used. The gap was usually set to $0.1 \mathrm{~mm}$ and $50 \mu \mathrm{l}$ were loaded unless otherwise mentioned. To prevent drying, a wet tissue was placed within the temperature chamber. Mineral oil was loaded surrounding the sample to prevent 
drying issues in longer measurements. Figure descriptions contain information on which method is used to prevent drying. For measuring crosslinking, time sweeps were performed. For UV crosslinking, the UV lamp from Thorlabs at $365 \mathrm{~nm}$ was used. After $1 \mathrm{~min}$ of measurement without light, the lamp was turned on to $68.7 \%$, which corresponds to $20 \mathrm{~mW} \mathrm{~cm}^{-}$ 2 in all measurements unless otherwise noted. For optimal results, the normal force was kept at zero to compensate for shrinking, which may occur during curing and lead to a negative normal force. Like this, the gel does not lose contact with the plate. Data points were collected each 5$10 \mathrm{~s}$. A shear strain of $0.5 \%$ or $1 \%$ (for the temp. sweeps) and frequency of $1 \mathrm{~Hz}$ or angular frequency of $10 \mathrm{rad} / \mathrm{s}$ were set. Curing was done at $22^{\circ} \mathrm{C}$ for most experiments or $25^{\circ} \mathrm{C}$ for mimicking volumetric printing with gelatin. For Temperature sweeps the temperature was decreased with a rate of about $1{ }^{\circ} \mathrm{C}$ per minute from $37^{\circ} \mathrm{C}$ to $4^{\circ} \mathrm{C}$.

Frequency and amplitude sweeps were performed to assess the mechanical behavior of the hydrogels after curing. Right after the UV crosslinking measurement, the frequency sweep was done in situ with an angular frequency of $0.1-100 \mathrm{rad} / \mathrm{s}$ and a strain of $0.5 \%$. In some cases, a subsequent amplitude sweep was performed with an angular frequency of $10 \mathrm{rad} / \mathrm{s}$ and a strain of $0.01-100 \%$ and in some cases even up to $1{ }^{\prime} 000 \%$. For the previously cured and swollen hydrogels, the PP10 was used as they had a smaller size, and the gap was set to have a normal force of around $0.1 \mathrm{~N}$.

\section{Permeability of Macroporous Hydrogels with FITC-Dextran}

The permeability of gels with FITC-dextran (500 kDa, Sigma-Aldrich) was studied similar as described elsewhere. ${ }^{7}$ In short, the gels were washed for three days at $4{ }^{\circ} \mathrm{C}$ in PBS till reaching a swelling equilibrium. Then, PBS was replaced with FITC-dextran and the sample was imaged after two days, when FITC-dextran reached equilibrium concentration.

\section{$\underline{\text { Turbidity Measurements }}$}

The turbidity was measured as the absorption at $405 \mathrm{~nm}$ with a plate reader (Spark M10, Tecan). Before curing, $40 \mu \mathrm{l}$ of the mixed precursor solution was loaded into a well of a 96 well plate. 
Gels cured in Teflon molds were punched with a $5 \mathrm{~mm}$ biopsy punch to fit into the plate and the turbidity measured with and without additional $100 \mu \mathrm{PBS}$ loaded into the well. Multiple well reads $(\mathrm{N}=5)$ were used as replicates with a border of $2 \mathrm{~mm}$.

\section{Volumetric (Bio)-Printing}

The volumetric printer Readily3D was used with the software Readily3D and Apparite. First, a laser dose test was conducted, in which defined spots were irradiated with varying laser intensity $(405 \mathrm{~nm})$ and irradiation time. For this, $500 \mu \mathrm{l}$ of precursor solution were loaded into a cuvette. The visible dots were noted in a table after the irradiation was done. A second test with a smaller range could then be conducted to more accurately determine the threshold needed for polymerization. For the print, commonly 3-4 $\mathrm{mL}$ of precursor solution was prepared and loaded into $18 \mathrm{~mm}$ diameter Pyrex vials. The refractive index of GelMA (1.37) was chosen and the peak-to-average ratio was usually set to $6: 1$. The optimal settings as calculated by the software were used.

\section{Imaging and Image Analysis}

The confocal microscopes Leica SP8 MP and Leica SP8 AOBS CARS were used for imaging. For imaging the whole volumetric print, a stereomicroscope (Leica Stereo) was used. All microscopes were used with the software LAS X. Images and videos during the volumetric printing process were captured with the integrated camera of the volumetric printer. Other images were taken with a smartphone. Image analysis was mostly performed in Fiji and is described in the following sections.

\section{$\underline{2 D \text { Fast Fourier Transform }}$}

2D-FFT is a common way to characterize the length scale of pores in bi-continuous structures. The analysis was done with a MATLAB code, which can be found in the supplementary information. In short, an image is read and converted to greyscale. Then the 2D-FFT of the image multiplied by a hamming window is taken. Further, radial binning is performed to show the radial intensity of the FFT (RIFFT). The length scale was then calculated as 1/q. 


\section{Porosity Analysis}

The threshold was set by Otsu's method and the area fraction was measured. 3 images $(\mathrm{N}=3)$ in different locations of the same z-stack were analyzed. Analyses where the background signal was too high were not included in the results

\section{Cell Area Quantification}

For the cell area quantification, maximum intensity projections (MIPs) of 3 x $100 \mu \mathrm{m}$ thick zstacks were analyzed per group. The threshold was manually set and kept the same for all groups within one experiment. The area was measured by the actin signal. The cell nuclei were then manually counted. The average cell area was then calculated by the total area divided by the cell number for each z-stack.

\section{Statistical Analysis}

Statistical analysis was performed in GraphPad Prism 8.2.0, except for the slope analysis, which was performed in Excel. Ordinary one-way or two-way analysis of variance (ANOVA) were performed depending on the number of variables. They were followed by Sidak's or Tukey's test for multiple comparisons for two or more groups respectively. If the standard deviations did not appear equal among groups, the Brown-Forsynthe and Welch ANOVA test was followed by Dunnett's multiple comparison test was applied. In the two-way ANOVA, only simple effects within rows and columns analyzed. Welch's t test was used when there were only two groups. The applied test is noted for each result. P values less than 0.05 were considered significant and are mentioned in the figure captions.

\section{Acknowledgements}

This work was supported by the SNSF Spark Award (no. 190345, X.H.Q.). We thank Wanwan Qiu for chemical synthesis of the nPVA. We are also thankful for the support of Paul Delrot and Damien Loterie for volumetric printing. Further, we thank Alba Sicher for sharing her code for the FFT analysis. We also gratefully acknowledge ScopeM for their support and assistance in this work. 


\section{References}

1. Griffin, D. R.; Weaver, W. M.; Scumpia, P. O.; Di Carlo, D.; Segura, T., Accelerated wound healing by injectable microporous gel scaffolds assembled from annealed building blocks. Nat Mater 2015, 14 (7), 737-44.

2. Stachowiak, A. N.; Bershteyn, A.; Tzatzalos, E.; Irvine, D. J., Bioactive Hydrogels with an Ordered Cellular Structure Combine Interconnected Macroporosity and Robust Mechanical Properties. Advanced Materials 2005, 17 (4), 399-403.

3. Nih, L. R.; Sideris, E.; Carmichael, S. T.; Segura, T., Injection of Microporous Annealing Particle (MAP) Hydrogels in the Stroke Cavity Reduces Gliosis and Inflammation and Promotes NPC Migration to the Lesion. Adv Mater 2017, 29 (32).

4. Sun, Q.; Gu, Y.; Zhang, W.; Dziopa, L.; Zilberberg, J.; Lee, W., Ex vivo 3D osteocyte network construction with primary murine bone cells. Bone Research 2015, 3 (1), 15026.

5. Sun, Q.; Choudhary, S.; Mannion, C.; Kissin, Y.; Zilberberg, J.; Lee, W. Y., Ex vivo construction of human primary 3D-networked osteocytes. Bone 2017, 105, 245-252.

6. Huebsch, N.; Lippens, E.; Lee, K.; Mehta, M.; Koshy, S. T.; Darnell, M. C.; Desai, R. M.; Madl, C. M.; Xu, M.; Zhao, X.; Chaudhuri, O.; Verbeke, C.; Kim, W. S.; Alim, K.; Mammoto, A.; Ingber, D. E.; Duda, G. N.; Mooney, D. J., Matrix elasticity of void-forming hydrogels controls transplantedstem-cell-mediated bone formation. Nat Mater 2015, 14 (12), 1269-77.

7. Broguiere, N.; Husch, A.; Palazzolo, G.; Bradke, F.; Madduri, S.; Zenobi-Wong, M., Macroporous hydrogels derived from aqueous dynamic phase separation. Biomaterials 2019, 200, 56-65.

8. Moroni, L.; Burdick, J. A.; Highley, C.; Lee, S. J.; Morimoto, Y.; Takeuchi, S.; Yoo, J. J., Biofabrication strategies for 3D in vitro models and regenerative medicine. Nat Rev Mater 2018, 3 (5), 21-37.

9. Gehlen, J.; Qiu, W.; Müller, R.; Qin, X.-H., Volumetric Tomographic 3D Bioprinting of Heterocellular Bone-like Tissues in Seconds. bioRxiv 2021, 2021.11.14.468504.

10. Zhang, J.; Wehrle, E.; Adamek, P.; Paul, G. R.; Qin, X.-H.; Rubert, M.; Müller, R., Optimization of mechanical stiffness and cell density of 3D bioprinted cell-laden scaffolds improves extracellular matrix mineralization and cellular organization for bone tissue engineering. Acta Biomaterialia 2020, 114, 307-322.

11. Zhang, J.; Eyisoylu, H.; Qin, X. H.; Rubert, M.; Muller, R., 3D bioprinting of graphene oxideincorporated cell-laden bone mimicking scaffolds for promoting scaffold fidelity, osteogenic differentiation and mineralization. Acta Biomater 2021, 121, 637-652.

12. Qin, X. H.; Wang, X.; Rottmar, M.; Nelson, B. J.; Maniura-Weber, K., Near-Infrared LightSensitive Polyvinyl Alcohol Hydrogel Photoresist for Spatiotemporal Control of Cell-Instructive 3D Microenvironments. Adv Mater 2018, 30 (10).

13. Moore, D. G.; Barbera, L.; Masania, K.; Studart, A. R., Three-dimensional printing of multicomponent glasses using phase-separating resins. Nat Mater 2020, 19 (2), 212-217.

14. Mayer, F.; Ryklin, D.; Wacker, I.; Curticean, R.; Čalkovský, M.; Niemeyer, A.; Dong, Z.; Levkin, P. A.; Gerthsen, D.; Schröder, R. R.; Wegener, M., 3D Two-Photon Microprinting of Nanoporous Architectures. Advanced Materials 2020, 32 (32), 2002044.

15. Dong, Z.; Cui, H.; Zhang, H.; Wang, F.; Zhan, X.; Mayer, F.; Nestler, B.; Wegener, M.; Levkin, P. A., 3D printing of inherently nanoporous polymers via polymerization-induced phase separation. Nature Communications 2021, 12 (1), 247.

16. Kleger, N.; Minas, C.; Bosshard, P.; Mattich, I.; Masania, K.; Studart, A. R., Hierarchical porous materials made by stereolithographic printing of photo-curable emulsions. Scientific Reports 2021, $11(1), 22316$.

17. Zaslavsky, B. Y., Aqueous two-phase partitioning: physical chemistry and bioanalytical applications. CRC press: 1994.

18. Bernal, P. N.; Delrot, P.; Loterie, D.; Li, Y.; Malda, J.; Moser, C.; Levato, R., Volumetric Bioprinting of Complex Living-Tissue Constructs within Seconds. Advanced Materials 2019, 31 (42), 1904209.

19. Kelly, B. E.; Bhattacharya, I.; Heidari, H.; Shusteff, M.; Spadaccini, C. M.; Taylor, H. K., Volumetric additive manufacturing via tomographic reconstruction. Science 2019, 363 (6431), 1075-1079. 
bioRxiv preprint doi: https://doi.org/10.1101/2022.01.29.478338; this version posted January 31, 2022. The copyright holder for this preprint (which was not certified by peer review) is the author/funder, who has granted bioRxiv a license to display the preprint in perpetuity. It is made available under aCC-BY-NC-ND 4.0 International license.

20. Wang, X.; Qin, X.-H.; Hu, C.; Terzopoulou, A.; Chen, X.-Z.; Huang, T.-Y.; Maniura-Weber, K.; Pané, S.; Nelson, B. J., 3D Printed Enzymatically Biodegradable Soft Helical Microswimmers. Advanced Functional Materials 2018, 28 (45).

21. Zaslavsky, B. Y.; Bagirov, T. O.; Borovskaya, A. A.; Gulaeva, N. D.; Miheeva, L. H.; Mahmudov, A. U.; Rodnikova, M. N., Structure of water as a key factor of phase separation in aqueous mixtures of two nonionic polymers. Polymer 1989, 30 (11), 2104-2111.

22. Song, S.-W.; Torkelson, J. M., Coarsening effects on the formation of microporous membranes produced via thermally induced phase separation of polystyrene-cyclohexanol solutions. Journal of Membrane Science 1995, 98 (3), 209-222.

23. Hirose, A.; Shimada, K.; Hayashi, C.; Nakanishi, H.; Norisuye, T.; Tran-Cong-Miyata, Q., Polymer networks with bicontinuous gradient morphologies resulting from the competition between phase separation and photopolymerization. Soft Matter 2016, 12 (6), 1820-1829.

24. Baudis, S.; Bomze, D.; Markovic, M.; Gruber, P.; Ovsianikov, A.; Liska, R., Modular material system for the microfabrication of biocompatible hydrogels based on thiol-ene-modified poly(vinyl alcohol). Journal of Polymer Science Part A: Polymer Chemistry 2016, 54 (13), 2060-2070.

25. Kimura, N.; Kawazoe, K.; Nakanishi, H.; Norisuye, T.; Tran-Cong-Miyata, Q., Influences of wetting and shrinkage on the phase separation process of polymer mixtures induced by photopolymerization. Soft Matter 2013, 9 (35), 8428-8437.

26. Butler, M. F.; Heppenstall-Butler, M., Phase separation in gelatin/dextran and gelatin/maltodextrin mixtures. Food Hydrocolloid 2003, 17 (6), 815-830.

27. Ouyang, L.; Armstrong, J. P. K.; Lin, Y.; Wojciechowski, J. P.; Lee-Reeves, C.; Hachim, D.; Zhou, K.; Burdick, J. A.; Stevens, M. M., Expanding and optimizing 3D bioprinting capabilities using complementary network bioinks. Science Advances 2020, 6 (38), eabc5529.

28. Qin, X.-H.; Labuda, K.; Chen, J.; Hruschka, V.; Khadem, A.; Liska, R.; Redl, H.; Slezak, P., Development of Synthetic Platelet-Activating Hydrogel Matrices to Induce Local Hemostasis. Advanced Functional Materials 2015, 25 (42), 6606-6617. 